\title{
El periodismo ciudadano. Análisis de opiniones de periodistas profesionales de España, Italia y Bélgica
}

\author{
Citizen Journalism. Analysis of opinions of journalists \\ from Spain, Italy and Belgium
}

\author{
Juan Carlos Suárez-Villegas / jcsuarez@us.es \\ https://orcid.org/0000-0002-2199-7028 \\ Universidad de Sevilla, España
}

\begin{abstract}
One of the most significant changes in journalism over the last decade has been the participation of citizens in the information process, establishing various forms of interaction with professional journalists and the media. This development has established a new journalistic culture based on dynamic interaction with the audience that improved information processes in various ways. However, this active role of the audience can not substantiate the so called citizen journalism, because it lacks the necessary conditions to ensure quality information that secures constructive and civilized public debate.
\end{abstract}

Key words: journalism, citizenship, interaction, democracy, information.

Resumen: Uno de los cambios más significativos en el periodismo de la última década ha sido la irrupción de los ciudadanos en el proceso informativo, estableciendo diversas formas de interacción con los profesionales de la información, con los medios de comunicación y también entre ellos. Esta novedad ha establecido una nueva cultura periodística donde la noticia adquiere una dimensión dinámica y en la que la propia interactividad con el público puede contribuir a alimentar distintos aspectos que también han entrado a formar parte de los medios de comunicación. Sin embargo, este papel activo del público no puede sustanciar el denominado periodismo ciudadano, pues adolece de las garantías necesarias de una información de calidad que asegure un debate público ordenado y constructivo.

Palabras clave: periodismo, ciudadanía, interactividad, democracia, información. 


\section{Introducción ${ }^{1}$}

El periodismo ciudadano es una etiqueta que alcanzó una gran popularidad en la primera década del siglo XXI, como una modalidad amateur de la profesión llamada a dar respuesta a la desconfianza generada por los medios de comunicación entre la sociedad. Con la inclusión de ciudadanos en el proceso informativo, se favorecía una mayor apertura en la configuración de la agenda pública, se contribuía a una mayor pluralidad de fuentes frente a los monopolios empresariales y se conseguía, en definitiva, dar un nuevo aire al periodismo, gracias en gran medida a las posibilidades abiertas por las nuevas tecnologías digitales.

Cualquier ciudadano con un teléfono móvil o una cámara podía ser testigo de un hecho noticioso y difundir ese material para el consumo del resto de la sociedad. Y no sólo videos y fotografías, sino también relatos desde el escenario de los hechos mediante los cuales suplir a los cronistas profesionales. Los propios medios de comunicación se han valido de este nuevo rol informativo del ciudadano en determinadas situaciones en las que era más fácil publicar una fotografía o una información remitida por un testigo que enviar a un equipo de profesionales al lugar.

En este sentido, las noticias de última hora de gran impacto, como atentados, desastres naturales o conflictos bélicos han sido una oportunidad idónea para que ciudadanos anónimos viesen sus contenidos publicados en primera plana en medios de alcance internacional. Los propios testigos de la tragedia de los atentados del metro de Londres en julio de 2005 subieron videos a Youtube y fotografías a Flickr, del mismo modo lo hicieron quienes atestiguaron la masacre del instituto de Virginia de 2007, el aterrizaje forzoso del avión en el río Hudson en 2009, el terremoto de Haití de 2010, las protestas ciudadanas en el norte de África y Oriente Próximo de 2011 y un sinfín de hechos acaecidos en los últimos años que corroboran esta relación entre medios y usuarios (Suárez-Villegas y Jiménez-Gómez, 2015).

No obstante, no todos los productos noticiosos en los que haya contribuido de una u otra forma un miembro del público pueden ser denominados periodismo ciudadano. Según la definición de Bowman y Willis (2003: 99),

1 Este artículo ha sido elaborado dentro del Proyecto del Plan Nacional de I+D+I del Ministerio de Economía y Competitividad del Gobierno de España con referencia CSO2011-26620: "Desafíos éticos en el periodismo digital. Análisis comparativo entre cinco países europeos" para el periodo 2012-2014. Con posterioridad será aceptada por dicho Ministerio la modificación de la investigación para llevarla a cabo en tres países en vez de cinco, justificado por la reducción del presupuesto solicitada para dicha investigación. 
podemos hablar de periodismo ciudadano cuando el usuario “juega un rol activo en la adquisición, reporte, análisis y diseminación de noticias e información"; es decir, cuando es el responsable último de recopilar, editar y distribuir la información mediante plataformas digitales autogestionadas, controlando de este modo todos los procesos productivos de la noticia.

Esta definición marca la diferencia con el periodismo participativo, en el cual la colaboración entre ciudadanos y periodistas se desarrolla en el marco profesional del medio. Por ejemplo, un video sobre un huracán enviado por un testigo a la redacción del medio y publicado en su versión digital sería una práctica enmarcada en el periodismo participativo. Ahora bien, si ese video es compartido directamente en una red social o un blog personal, se consideraría periodismo ciudadano.

Con el objeto de profundizar más en el concepto, podemos establecer hasta tres elementos clave en la naturaleza del periodismo ciudadano: la publicación abierta a partir de herramientas de bajo coste y fácil uso, la edición colaborativa con plataformas de interacción como chats, foros o redes sociales, y la distribución desintermediada de contenidos. Estas características nos llevan a una cultura informativa diferente basada en la interconexión permanente de los nodos de una extensa red descentralizada, donde se crean, recrean, consumen y comparten contenidos al margen de los cauces tradicionales de consumo. Es lo que Axel Bruns (2008) denominó como "produsage", haciendo referencia a la naturaleza dual del ciudadano digital que consume y produce a la vez información como un actor comunicativo más.

Las redes sociales son un buen ejemplo de esta práctica por sus características inherentes. Harrison y Barthel (2009: 174) argumentan que la "trascendencia de las redes sociales residen en su capacidad para que un volumen mayor de usuarios experimenten con una variedad más amplia y heterogénea de actividades creativas colaborativas", algo muy cercano al concepto de "inteligencia colectiva" propuesto por Malone et al. (2009), para referirse a esa actividad colaborativa a partir de un modelo de comunicación móvil, ubicuo y asincrónico, en el cual se confunden lo público y lo privado.

Las funciones y los usos de las redes sociales son numerosos y varían desde el entretenimiento hasta la expresión personal o los fines informativos. De hecho, en los últimos años ha sido patente cómo estas herramientas han ganado protagonismo como plataformas de consumo informativo hasta el punto de estar integradas en los medios de comunicación con botones, mediante los que compartir las noticias, incrementando de este modo el recorrido de los artículos más allá de la website del medio. También han sido fundamentales en la cobertura de hechos noticiosos de última hora. 
No en vano, Bruno (2011: 64) proponía el concepto de "efecto Twitter" para definir un fenómeno por el cual "he content circulating in the social media has become an integral part of today's newsgathering during major events. Real time tweets, amateur videos on YouTube and first-hand accounts on Facebook fill up the 'news-vacuum' that until a few years ago characterized the aftermath of major crisis events".

Esta dinámica colaborativa ocurre de manera simultánea a otras iniciativas adoptadas por los ciudadanos para ofrecer sus propias versiones de temas de interés. Se trata de otros formatos donde los ciudadanos ofrecen contenidos que pueden ser de interés para el resto de la sociedad, pero que son confeccionados al margen del sector profesional, aprovechando los cauces habilitados por las tecnologías de autoedición. Ya no se trata tan sólo de contenidos difundidos a través de redes sociales, sino de espacios donde existe una clara motivación informativa desarrollada a partir de unos criterios similares a los periodísticos.

Por ejemplo, los blogs o bitácoras se popularizaron a mediados de la década de 1990 como una herramienta de fácil uso que permitía publicar opiniones e información de forma descentralizada, además de crear redes de usuarios con los que compartir artículos y datos, lo cual suponía una diferenciación clara respecto al modelo tradicional de comunicación pública. De acuerdo con Singer (2005), "rather than acting as gatekeepers, bloggers see themselves as serving a nearly opposite function: Providing a venue in which whatever anyone in the world knows or thinks can receive a hearing and then be publicly debated. Blogs are, if you like, the 'marketplace of ideas' with a vengeance".

Paulatinamente, los blogs ciudadanos se han ido incorporando a la oferta de los medios de comunicación, dado su fuerte impacto en la opinión pública en modelos exitosos como el implementado por The Huffington Post, un medio nativo de origen norteamericano en el cual buena parte de los contenidos son generados por blogueros.

Además de redes sociales y blogs, a comienzos de siglo surgieron algunas plataformas informativas gestionadas exclusivamente por ciudadanos, como foros de opinión y medios amateurs. No obstante, es preciso diferenciar entre formatos íntegramente desarrollados por ciudadanos de aquellos en los que existía una gestión profesional. Por ejemplo, el sitio coreano de noticia OhMyNews ha sido tradicionalmente utilizado para ilustrar el fenómeno del periodismo ciudadano; sin embargo, su funcionamiento dependía del trabajo de un grupo de periodistas que editaban las contribuciones de los ciudadanos.

El sitio web fue fundado en el año 2000 con una redacción de periodistas reducida que se encargaba de verificar mínimamente las historias remitidas 
por miles de usuarios. De hecho, el éxito del periódico, que incluso abrió una versión japonesa a la postre fallida en 2006, fue la razón de su propio fracaso, ya que los profesionales del medio eran incapaces de gestionar el volumen ingente de informaciones enviadas por los periodistas ciudadanos, provocando un cambio de formato y filosofía en 2010.

Como el caso de OhMyNews, numerosas plataformas colaborativas de ciudadanos y periodistas surgieron por todo el mundo. Incluso grandes medios de comunicación incluyeron secciones donde se invitaba a los usuarios a participar con contenidos elaborados. Por ejemplo, en España, el diario El País inauguró en 2007 la sección Yo Periodista; sin embargo, quedaría inhabilitada tan sólo tres años después con resultados cuestionables (Suárez-Villegas y Jiménez-Gómez, 2015).

Ahora bien, ¿cuál es la opinión de los propios periodistas acerca del fenómeno denominado "periodismo ciudadano"? Dentro de un proyecto de $\mathrm{I}+\mathrm{D}+\mathrm{I}$ sobre la ética en el periodismo digital en tres países europeos: España, Italia y Bélgica, hemos pretendido profundizar en la opinión de los profesionales respecto a este asunto. $Y$ a modo preliminar de las conclusiones defendidas en el presente artículo, podemos adelantar que existen claras discrepancias entre los profesionales acerca del valor que habría que concederle a las aportaciones del público. La mayoría advierte del riesgo de calificar esta actividad como periodismo ciudadano, pues se presta a que sea confundida con la actividad realizada por los profesionales.

Por esta razón, más que periodismo, entendido como un ejercicio reglado y que presta un servicio público, se considera más oportuno calificar estas prácticas de comunicación interactiva entre ciudadanos privados, donde prevalece su perspectiva subjetiva y la ausencia de garantías derivadas de un protocolo de diligencia profesional. Por ello, tales aportaciones más bien deberían ser consideradas como pistas o indicios informativos, que una vez verificados por otras fuentes y debidamente contextualizadas, pueden adquirir valor informativo.

Por tanto, los periodistas muestran ciertas cautelas hacia esta forma de "periodismo ciudadano" como una vía de vehicular la información. En cambio, una vez aclarado que de estas aportaciones de los usuarios no se puede fundar el legítimo derecho a garantizar el derecho a ser informado, así como un debate público estructurado, sí se muestran partidarios de aceptar el hecho de que los ciudadanos se hayan convertido en agentes fundamentales en el proceso informativo a partir del desempeño de distintas funciones. Así pues, desde una perspectiva general, los profesionales no aceptan el periodismo ciudadano como tal pero sí el periodismo con los ciudadanos. 
Y es que con el desarrollo de internet y los usos sociales a éste adheridos, se ha pasado de una cultura en la que el periodista "informaba" al público de un modo unidireccional a otra donde el periodista se comunica con el público organizado en comunidades virtuales y con un rol activo en la construcción de la noticia, ya sea aportando datos, temas de interés o contribuyendo a la difusión de la misma. Es decir, esta actividad conjunta se desarrolla dentro de un nuevo ecosistema informativo más horizontal en el cual el público interviene en las distintas partes del proceso informativo a través del uso de las nuevas tecnologías. El límite se impone, no obstante, en la necesidad de una pertinente verificación de estas aportaciones por parte de los profesionales.

Desde la doctrina constitucional se ha subrayado con insistencia la diferencia entre el derecho a informar, como un derecho de emisores, del derecho a ser informado, como un derecho del público, siendo ambos distintos tanto en sus límites como en sus deberes. Mientras que el primero sería ejercido sin mayores exigencias por cualquier ciudadano que narra un episodio desde su punto de vista o expresa su opinión sobre unos hechos, el segundo constituye un derecho que vertebra el conocimiento y debate público de los asuntos que conciernen al conjunto de la ciudadanía. Por tanto, se trata de un derecho esencial donde se asienta la sociedad democrática y que requiere una actividad organizada e institucionalizada que vele por la correcta administración de dicho derecho.

Sería en este derecho en el que se fundamenta la libertad de prensa, por la cual los periodistas profesionales detentan una función cualificada en su ejercicio, y asumen una especial responsabilidad derivada de las cualidades exigidas a dicha información: veracidad, independencia, pluralidad e interés público. Estas cualidades de la información exigen por parte del profesional, asimismo, una dimensión moral que se cimienta en la honestidad, entendida como la prioridad de su compromiso con la ciudadanía por encima de cualquier otro tipo de intereses.

En el momento actual donde profesionales de la información y ciudadanos han ocupado posiciones más horizontales en el debate público, se ha quebrado la preponderancia del profesional como gatekeeper de la información, pues ésta puede también difundirse por otros cauces (Dahlgren, 2016). Por otro lado, los usuarios se han hecho presentes en las redacciones a través de sus contribuciones en el proceso informativo, de tal manera que hoy en día la noticia se ha convertido, en cierto modo, en un trabajo colaborativo. Por eso, ante la dicotomía entre derecho a informar y derecho a ser informado, surge una nueva dimensión que sería el derecho de los ciudadanos a participar en el proceso informativo, pero no sólo como un derecho de emisores, sino como agentes que colaboran con los profesionales, cada uno con sus distintas funciones. 
Esta nueva cultura ha convertido la noticia en un relato que adquiere una posterior dimensión a partir del propio uso social que se hace de ella, por lo cual el periodista no sólo debe informar, sino que ha de asumir una responsabilidad más directa sobre los efectos que dichas noticias causen sobre los ciudadanos. Por tanto, si bien los grandes medios siguen dominando el panorama comunicativo, cada vez se producirá una mayor interacción entre medios y comunidades virtuales, donde la actividad informativa será complementada con las aportaciones del público.

Este recurso a contenidos remitidos por ciudadanos no es visto, sin embargo, por todos los profesionales como algo positivo. En una investigación conducida por Anden-Papadopoulos y Pantti (2011), se establecen hasta tres posturas hacia dicha colaboración: de resistencia, donde se incide en las capacidades y habilidades de los profesionales frente a los amateurs; de resignación, donde se reconoce cierta presión para incorporar este tipo de contenidos por la nueva lógica del periodismo digital; y de aceptación, por la que se sugiere la adopción de un nuevo espíritu más abierto al contexto actual donde prime la participación, la transparencia y una cultura mediática más horizontal.

En el estudio realizado por Harro-Loit (2015), donde se cuestiona a periodistas profesionales acerca de un valor tan arraigado como la responsabilidad o accountability, la conclusión extraída es que existe cierta desconfianza hacia todo contenido que no es recabado directamente por el periodista según sus criterios y procedimientos habituales. Tal y como se establece al final del artículo, esa visión tradicional del proceso de construcción de la noticia, conduce a los periodistas a adoptar buenas prácticas deontológicas basado en sus principios y valores profesionales.

El ámbito del fotoperiodismo, por ejemplo, es uno de los que más ha sido comprometido por la irrupción de ciudadanos en la provisión de material audiovisual, hasta el punto de que éstos son más requeridos que los propios profesionales (Anden-Papadopoulos y Pantti, 2011). En el estudio de Mortensen (2014), donde se compara la ética de sendos actores, se refleja la confusión reinante a este respecto y la confrontación experimentada en torno a quién desarrolla con mayor responsabilidad y rigurosidad su labor. En cualquier caso, es un hecho que prácticamente la totalidad de los fotoperiodistas ciudadanos, aunque cuenten con experiencia profesional, no son remunerados por los contenidos.

En conclusión, y tal y como se desprende de la mayoría de los estudios referidos, no se trata de alentar el optimismo de que los individuos se conviertan en medios de comunicación en sí mismos (Gillmor, 2006; Bowman y Willis, 2003), sino de establecer vías para reforzar el derecho a ser informado 
con la participación de los ciudadanos en dicho proceso. De esta manera, ambas fases previas quedarían integradas en esta nueva dimensión del derecho informativo de la ciudadanía a ser informado.

\section{Metodología}

Este trabajo se enmarca dentro del proyecto de Investigación, Desarrollo e Innovación del Ministerio de Economía y Competitividad titulado "Desafíos éticos del periodismo digital. Un estudio comparativo entre tres países europeos: España, Italia y Bélgica”. El presente artículo describe las actitudes manifestadas por los periodistas digitales de estos tres países ante el denominado periodismo ciudadano.

Dicha investigación se desarrolló en dos fases: una cuantitativa y otra cualitativa. La primera fase consistió en formular una serie de preguntas con respuestas cerradas sobre cuestiones relativas a los cambios que las nuevas tecnologías han propiciado en la cultura profesional, en su dimensión ética fundamentalmente. Entre otras conclusiones, se puso de manifiesto una atomización del trabajo periodístico, con nuevas fórmulas de teletrabajo y de periodismo local más fáciles de gestionar y financiar gracias a las nuevas tecnologías. En esta fase también se observó cómo los ciudadanos se han convertido en agentes más activos en el proceso de construcción de la noticia en un nuevo ecosistema informativo.

En este trabajo se presenta una síntesis de los datos cuantitativos y de la fase cualitativa de dicho estudio. De manera más específica nos centramos en la pregunta sobre "¿qué valoración le merece el denominado periodismo ciudadano?", dentro de un conjunto más amplio de cuestiones destinadas a analizar las novedades aportadas por internet a la dinámica informativa, referidas a la relación entre profesionales y público. Se trataba de un modelo de entrevista semiestructurada realizada a lo largo de 2014 a más de setenta periodistas de estos tres países.

Los perfiles profesionales de los participantes eran diversos, desde redactores hasta responsables de medios, con una edad media de 36 años de todos los participantes, y con un porcentaje del $58 \%$ de hombres entrevistados frente al $42 \%$ de mujeres. En cada país se realizaron 24 entrevistas, de las cuales fueron elegidas veinte por país para llevar a cabo el estudio comparativo.

A través de diversas cuestiones enfocadas a registrar el valor periodístico que atribuían a las aportaciones de los ciudadanos para vigorizar la opinión pública, una de estas preguntas perseguía conocer qué valor le atribuían al determinado periodismo ciudadano; es decir, si las aportaciones de los ciu- 
dadanos a través de diferentes formatos digitales podían ser una alternativa informativa a la función realizada por los medios de comunicación. De qué manera entender y conciliar las aportaciones de los ciudadanos y la función de los periodistas profesionales dentro del nuevo ecosistema digital.

Ante un universo de periodistas desconocido, algunos autores consideran justificable entrevistar en profundidad a una veintena de sujetos, ya que a partir de dicho número se produce lo que se conoce como saturación; esto es, que las entrevistas adicionales no producen "nuevas visiones en las variables claves” (Hughes, 2012). El artículo posee, por tanto, un carácter cualitativo y resulta útil en la medida en que contribuye a detectar nuevas tendencias acerca de la compleja relación entre periodismo, ciudadanía y democracia (Suárez-Villegas y Jiménez-Gómez, 2015).

\section{Resultados}

En primer lugar, ofreceremos algunos datos correspondientes a la parte cuantitativa de la investigación que refleja la opinión de los profesionales sobre la participación de los ciudadanos en los procesos informativos de manera positiva. En cambio, en la parte cualitativa se advierte que esta nueva dinámica participativa no parece que pueda fundamentar el denominado periodismo ciudadano como una forma espontánea de interacción informativa de la cual surja una opinión pública crítica y organizada.

En la encuesta, en relación con la cuestión de cómo valora la aportación de los ciudadanos a la actividad informativa, el $42,3 \%$ de los encuestados italianos afirma que los ciudadanos contribuyen efectivamente a la búsqueda de información en su medio de comunicación, frente a un 38,5\% de los españoles y un $36,7 \%$ de los belgas. Un $62,6 \%$ de los italianos, un $42,3 \%$ de los españoles, un $34,6 \%$ de los belgas menciona su papel en la verificación de informaciones. Y 60,2\% de los italianos, 35,5\% de los españoles y 30,7\% belgas se refieren a su participación en la contextualización de las mismas.

Más allá de esta constatación de la importancia de la participación de la ciudadanía en los medios actuales, los encuestados expresan su opinión sobre el significado ético de dicha participación, pensando especialmente en los comentarios que se publican en los medios digitales o en las versiones digitales de los medios profesionales:

Un 30,3\% de los profesionales españoles consultados, 53,9\% de los belgas y $58,4 \%$ de los italianos consideran la participación de los ciudadanos como una muestra de democracia y de diálogo racional, libre y plural. Sin embargo, 29,4\% de los españoles, 53,9\% de los belgas y 39,6\% de los italianos 
muestran su acuerdo con las afirmaciones de que los comentarios tienden a ser disparatados y/o acalorados.

En un escenario propositivo, con el fin de conseguir una participación ciudadana en los medios más acorde con el ideal de una sociedad democrática, $43,1 \%$, de los encuestados españoles, $61,5 \%$ de los belgas y $62,5 \%$ de los italianos se muestran partidarios de no permitir los comentarios anónimos. Un $52,5 \%$ de los españoles, $61,6 \%$ de los belgas y $68,7 \%$ de los italianos consideran que es necesario filtrar los comentarios para evitar aquellos que puedan ser calumniosos, ofensivos o vejatorios; mientras que $42,4 \%$ de los españoles, $53,9 \%$ de los belgas y $50 \%$ de los italianos abogan por exigir a los usuarios registrarse para poder participar con sus comentarios.

En la parte cualitativa, son más patentes las convicciones éticas de los periodistas sobre el interés creciente de una cultura profesional diferente y más participativa, fruto de una interactividad constante con los ciudadanos, aun sin confundir los roles de los periodistas y usuarios; esto también se puso de manifiesto en los resultados de las entrevistas en profundidad realizadas. En líneas generales, todos coinciden en que el periodismo ha cambiado en sus formas aunque no en su esencia, pues el periodismo sigue enraizado en la búsqueda de la veracidad, entendida como un método profesional basado en la comprobación de fuentes, la pluralidad de perspectivas y la distinción entre hechos y valoraciones, entre otras medidas.

En el ámbito del periodismo digital, donde esta tarea se ha tornado cada vez más colaborativa entre periodistas y ciudadanos a raíz de las posibilidades abiertas por las nuevas tecnologías, el reto consiste en preservar ese método profesional, al mismo tiempo que se da entrada a los usuarios al proceso informativo siguiendo una fórmula por la cual sendas esferas permanezcan relativamente separadas.

De hecho, la mayoría de los periodistas entrevistados muestra su rechazo a denominar la labor auxiliar y esporádica que ejercen los ciudadanos a través del soporte digital como periodismo ciudadano. De manera bastante elocuente, uno de los entrevistados nos ilustra su postura:

Yo niego la mayor. No creo que exista el periodismo ciudadano. Salvo que usted me permita a mí que cuando yo cambie una bombilla de una lámpara de mi casa, yo soy electricista ciudadano. $\mathrm{O}$ si curo una herida de mi hijo pequeño, soy médico ciudadano. Permítame esta comparación. Yo creo que el periodismo ciudadano no existe. El periodismo es uno, se escribe con mayúsculas y tiene la misma capacidad y los mismos valores éticos que hace 50 años. Es un trabajo que se basa en el contraste de la información y en el basamento de pruebas reales que determina unas afirmaciones que hace el periodista. Y eso no lo hace el periodismo ciudadano. Así que no confundamos (reportero español, 62 años). 
Ahora bien, negar el periodismo ciudadano no es negar la necesidad de colaborar con los ciudadanos y abrirse a que sus aportaciones pueden formar parte del material con el que trabajan los periodistas para hacer la información. Hoy en día cualquier persona cuenta con un teléfono móvil que graba videos o realiza fotografías de una calidad aceptable que pueden devenir en documentos gráficos de gran valor periodístico.

La función testimonial desempeñada por ciudadanos que asisten a hechos de trascendencia como un conflicto armado de difícil acceso o un acontecimiento de última hora al que aún no se han desplazado periodistas, tal y como se ha demostrado en multitud de circunstancias en los últimos años en las cuales estos contenidos han abierto telediarios o aparecido en portadas de diarios internacionales, es una de las posibilidades abiertas por una colaboración estrecha entre periodistas y usuarios que se practica con asiduidad.

Como referíamos anteriormente, el periodismo adquiere hoy más que nunca una cierta dimensión colaborativa que presenta numerosos riesgos éticos. El periodista no puede renunciar a asumir con seriedad y rigor su labor profesional, pues quien reproduce sin más lo que le llega de un tercero, aunque lo compruebe y sea cierto, tendrá el deber de consultar a la otra parte e informar desde una visión completa de los acontecimientos. A este respecto, una de las entrevistadas comenta a propósito de una cuestión de actualidad, como son los desahucios:

Yo qué sé, una cosa muy en boga últimamente, un desahucio. A mí que alguien de la Plataforma de Afectados por la Hipoteca me envíe un vídeo de un desahucio, es una información que me es útil, pero no es periodismo. No sé cuál es la postura de la inmobiliaria o del banco o... No lo sé, no lo sé. Pero entiendo que la PAH no tiene obligación de enviarme ese contrapunto. Ellos te dan su información, lo que les afecta a ellos. No sé si es un ejemplo muy feliz, pero, yo qué sé... O alguien a quien le expropian el piso o el terreno: "El Ayuntamiento o el Ministerio me expropian este terreno que es mío, no sé qué, no sé cuál...”. Y te envía un vídeo o una nota diciendo que es una injusticia y que no sé qué no sé cuántos... Bueno, eso no es periodismo ciudadano. Es un testimonio de un ciudadano que tú le has de dar la importancia que tú le quieras dar, pero, oye, ¿qué pasa con esto? Si tú preguntas qué pasa con esto, inmediatamente necesitas otra fuente (reportera española, 53 años).

El periodista cuenta hoy con más recursos adicionales para construir su pieza informativa, pero ésta no puede basarse exclusivamente en elementos gráficos o manifestaciones descontextualizadas. Tal y como argumenta uno de los periodistas entrevistados, "no puede confundir aquello que llama la atención con lo que es el núcleo de un problema. Sólo cuando entiende de lo que habla, de lo que está pasando, podrá informar con conocimiento de causa" (reportero italiano, 35 años). Es decir, el periodista que está esperando 
las piezas que llegan a la redacción sin adentrarse en el terreno donde se producen los hechos sólo podrá repetir lo que le dicen otros, pero no dispondrá de criterios interpretativos para enfocar la noticia desde el interés de los ciudadanos; por lo tanto, se puede convertir en un simple vocero de una de las partes.

Por eso, la mayoría de los periodistas entrevistados considera que las aportaciones remitidas por los ciudadanos a los medios, como fotos, videos sobre un determinado episodio, testimonios de sus protagonistas o cualquier otro elemento de interés informativo son materiales que pueden resultar valiosos una vez verificados y contextualizados en el relato de los acontecimientos, pero convertirlo en noticias por disponer de dichos recursos sin entender su significado es una manera de falta a la verdad, con el riesgo de dar una imagen desproporcionada a favor de una de las partes, por lo que se pueda incurrir en una burda manipulación por rentabilizar el beneficio generado por imágenes sensacionalistas.

Como declara uno de los entrevistados, "una agenda creada en exclusiva por medios de periodismo ciudadano sería tan peligrosa como una agenda de profesionales que descuida la voz de los medios sociales de la Web". Por eso, prefiere optar por el término de "información ciudadana” en vez de periodismo ciudadano, pues, a su juicio, "me parece muy positiva y enriquecedora la aportación que los ciudadanos pueden hacer al periodismo como testigos, aportando elementos de análisis, ampliando la agenda y colaborando con la redacción de los medios, pero considero que la gestión de sus derechos y de los contenidos que aportan no son las apropiadas" (reportero belga, 32 años).

Por su parte, los colegas belgas consideran que la denominación "periodismo ciudadano” resulta delicada, pues podría dar a entender que todo el mundo puede ser periodista. Apuntan que es necesario especificar y diferenciar al periodista profesional de quien no lo es, pues el periodismo ciudadano no da garantía al público de su credibilidad y respeto a la deontología profesional. En primer lugar, sostienen que también es imprescindible conocer qué clase de ciudadano es el que está colaborando; si se trata de un testigo de hechos, un experto en el tema, un colaborador de prensa habitual, un usuario anónimo que comparte información como fuente del periodista...

Algunos profesionales belgas comentan que estos contenidos remitidos por ciudadanos, cada vez más numerosos y que llegan directamente a las redacciones sin necesidad de buscarlos, son nuevas fuentes informativas con las cuales hay que adoptar las mismas medidas verificadoras. Al fin y al cabo, continúan; el periodismo es una profesión que realiza una actividad de mayor envergadura y los hechos sólo son noticias cuando son comprobados y contextualizados al completo y en relación con la actualidad. 
La característica del profesional no es sólo lo que dice, sino el conocimiento que tiene sobre lo que dice y el modo como sabe contarla para poner de relieve su relación con el conjunto de la realidad que afecta a los individuos. Así, uno de los entrevistados apunta que "gracias al periodismo ciudadano se escapan menos noticias que antes, pero por otro lado se hace más complicado contrastar la noticia, contextualizarla y conocer su procedencia” (reportero español, 38 años).

Otros entrevistados, en cambio, realizan una valoración negativa de este tipo de periodismo, pues sólo contribuye a confundir al ciudadano sobre qué debe ser tomado como noticia. De hecho, algunos sostienen que para un medio digital es más difícil lograr una entrevista que para un periódico impreso, pues "se asocia con un periodismo de menor calidad y seriedad". Otro peligro que han destacado los entrevistados a raíz del periodismo ciudadano es que los medios de comunicación están dejando de enviar a los periodistas para cubrir los acontecimientos sobre el terreno, ya que obtienen material gráfico y audiovisual de manera gratuita a través de los ciudadanos.

Esta confianza en los testimonios de los ciudadanos desvirtúa en cierto modo el proceso de construcción de la noticia, en la medida en que se deberían obtener sobre el terreno por parte de un periodista profesional, capaz de apreciar los hechos desde un enfoque más imparcial y plural. Como señalan varios periodistas incluidos en el estudio, se corre el peligro de perder el pulso de los acontecimientos y que éstos se vean reconstruidos por los periodistas a partir de fragmentos de testimonios que no pueden comprobar directamente respecto a los hechos.

Se desprende, por tanto, de las opiniones de los periodistas, que la comunicación con el público es un valor añadido para la actividad informativa, pues puede complementar las noticias con elementos que pueden ser útiles; pero esto no es lo mismo que confundir el periodismo con la comunicación del público, pues se perdería la esencia del periodismo como un trabajo profesional de informar con conocimiento veraz de los hechos y con un compromiso de asumir una función de atender al interés público. Además, el periodista no debe optar por que le llegue la noticia, lo cual le concede una posición de preeminencia a quien la remite, sino que ha de buscarla, comprobarla y relacionarla con una visión completa de los acontecimientos.

No obstante, la mayoría de los entrevistados considera que la posibilidad de interacción con el ciudadano es un avance democrático, pues el público puede expresarse y en algunos casos incluso llegar a completar la información; por lo tanto, lo estima positivo. Una de las periodistas entrevistadas afirma incluso que hay una relativa representatividad de la ciudadanía en 
internet; sin embargo, según su opinión, sería utópico concederle el valor de la opinión pública, la cual requiere procesos de confrontación que conduzcan a posiciones consensuadas. Además, no todos los ciudadanos están representados en la red y no todos lo hacen en el mismo grado y capacidad, por lo que dentro de la red también pueden producirse fenómenos de desigualdad informativa que no deberían ser obviados.

Por otro lado, numerosos periodistas comentan que los foros digitales y espacios de comentarios no son verdaderos espacios de debate e intercambio de opiniones y argumentos, sino espacios adicionales de expresión personal. En relación con los comentarios que los ciudadanos adhieren a las noticias, la mayoría de los entrevistados advierte que tienen más un carácter de desahogo o reacción emotiva que de discurso racional.

Esto surge de la percepción que, por un lado, los comentarios que los usuarios generan no tienen contenido interesante o constructivo para añadir a la noticia; y, por el otro, y a nivel ético y deontológico, esos comentarios no son adecuados en un porcentaje muy elevado de los casos, lo cual resta calidad al medio digital, pues con frecuencia se publican contenidos de carácter insultante o sin relación al contenido de la noticia.

Otro de los periodistas comenta que la participación de las audiencias "es algo reciente y que aún le queda un camino por recorrer para que pueda realmente contribuir a la calidad el periodismo”. A su juicio, habría que aspirar al modelo que se está implantando en Estados Unidos, dirigido a que esa participación convoque voces de expertos en determinados temas para que comenten o soliciten un cambio en la noticia, con el ánimo de aumentar la calidad de la información. En este mismo sentido, algunos de los entrevistados, en su mayor parte belgas, sostienen que la interacción de las audiencias con el medio no es algo que posea relevancia a la hora de hacer periodismo: "los medios ofrecen la oportunidad porque no hacerlo estaría mal visto pero por lo general no se tienen en cuenta los comentarios ni opiniones de los usuarios, salvo en contadas excepciones" (reportero belga, 39 años).

De hecho, en Bélgica hay determinadas noticias en las que los comentarios permanecen cerrados, por ejemplo, en aquellas donde se tratan temas como la inmigración, el Islam y la homosexualidad. La visión general es que los medios son responsables de lo que publiquen y deben mantener la ética y la deontología tanto en sus contenidos como en los comentarios que los usuarios publiquen. Por ello, recalcan la importancia de regular la interacción y las reglas de participación, para que esto pueda contribuir a desarrollar un espacio más democrático. 
A nivel de contenido, los periodistas tampoco consideran los comentarios interesantes, salvo algunas excepciones en las que el usuario corrige algún término o idea desde una perspectiva experta. Respecto a la interactividad, señalan que no es resolutivo, pues no hay una interacción con el medio, sino entre los propios participantes. Coinciden en que uno de los pocos aspectos interesantes y prácticos es a nivel económico, pues, de acuerdo con las cifras de participación y tráfico generado por los usuarios en los medios, se calculan las tarifas publicitarias. Asimismo, señalan que las contribuciones de los usuarios tienen un impacto en el contenido del periodismo web, donde cada vez se demanda más participación y contenidos ligeros y de entretenimiento.

Los periodistas italianos, en línea general, mantienen una postura positiva acerca de la participación de los ciudadanos, pues además de ofrecer indicios sobre los cuales rastrear noticias, principalmente, transmite perspectivas heterogéneas sobre la propia actualidad. Tal y como sostiene uno de los periodistas,

es un pulso que conviene mantener para realizar un periodismo que vaya con las preocupaciones de la gente. De lo contrario, el periodismo puede ser una labor casi oficialista y establecer un escalón entre el discurso mediático y las preocupaciones de la ciudadanía. Conviene rellenar este espacio con historias que engarcen los macrodiscursos sociales con la intrahistoria de la gente. El periodista a fin de cuentas realiza una función esencial para la pervivencia de la democrática, por lo que debería atender a la voz de los intereses de los ciudadanos, si bien deberá hacerlo con el rigor que exige un tratamiento profesional (reportero italiano, 33 años).

En otras palabras, las quejas y denuncias de los ciudadanos necesitan desarrollar la punta de lanza que inspira una auténtica investigación periodística, y conectarla como parte de una información de interés público. En este sentido, ambas partes contribuyen a reforzar la función democrática de la prensa. Pero todos los periodistas insisten en que es una herramienta, un nuevo modo de estar en la "calle virtual", pero no es periodismo. Alertan que también en las calles se lanzan alarmas y noticias falsas con las que el periodista deber ser muy cauto a la hora de investigarlas, antes de hacerse eco de ellas, pues puede estar contribuyendo a sobredimensionar episodios alentados por ciertos grupos que buscan protagonismo en los medios.

Por otro lado, atendiendo a la distinción con la que iniciamos este artículo y siguiendo las opiniones de los entrevistados, no debe olvidarse que los ciudadanos se expresan sin estar sujetos a un marco de principios o deberes que garanticen la calidad informativa, por lo que prima su perspectiva subjetiva y, en ocasiones, poco ética. Por eso, el periodista, tal y como sostienen varios profesionales, ha de ser un filtro para interpretar el interés que pueda haber en determinadas informaciones ciudadanas, para rastrear y comprobar elementos que sí sean pertinentes para la construcción de las noticias. 
Otro de los periodistas entrevistados considera que "en cualquier caso no se sabe qué será el futuro y cómo se organizará estas contribuciones de los ciudadanos" (reportero belga, 44 años), por lo que considera que la única manera de evitar cualquier atisbo de comparación sería demostrando la diferencia que marca un trabajo profesional. "La ética, la formación, el rigor y el compromiso se antojan como cualidades indispensables para acreditar la labor del profesional y despejar cualquier duda acerca de este debate" (reportero belga, 53 años).

Pero los periodistas pierden la razón cuando se dedican a hacer lo mismo que los ciudadanos, noticias rebotadas o reportajes que se reproducen sin entender bien del tema que están tratando. Es decir, tal y como apunta uno de los entrevistados, "el periodista sólo será respetado si conserva la determinación de validar el rigor de la información y desarrolla otras actividades investigadoras para ofrecerle a los ciudadanos perspectivas más amplias de los acontecimientos" (reportero belga, 37 años).

\section{Discusión}

Los cambios desarrollados en el entorno periodístico digital en los últimos años a partir de la introducción de nuevas herramientas comunicativas y de la incorporación de ciudadanos en el proceso de construcción de la noticia, ha removido los cimientos de la profesión como nunca había ocurrido antes. La proliferación de fuentes alternativas de información en internet, donde el consumo se descentraliza y los medios de comunicación tradicionales pierden el monopolio del relato de la actualidad, ha propiciado el surgimiento de numerosos retos que afecta de forma directa la labor de los propios periodistas.

Quizás uno de esos retos que mayor atención académica y en el sector profesional ha despertado ha sido el concerniente al denominado periodismo ciudadano, una etiqueta bajo la cual se engloban aquellas prácticas donde usuarios no profesionales desempeñan una función informativa más o menos relevante, aprovechando las oportunidades brindadas por las nuevas herramientas tecnológicas y las plataformas digitales de comunicación. Desde la popularización de este concepto ya en el siglo XXI, el debate suscitado ha sido especialmente intenso y ha despertado posiciones encontradas dentro del sector periodístico.

El afán de este artículo era precisamente calibrar la opinión de periodistas de tres países diferentes sobre el periodismo ciudadano, conociendo los argumentos a favor o en contra de esta práctica. Tal y como hemos podido comprobar en el apartado de resultados, la percepción general es que el 
hecho de que una persona grabe en directo un determinado acontecimiento o escriba un artículo sobre un tema concreto, no lo hace periodista incluso si el alcance de su contenido es masivo. La mayoría de los profesionales entrevistados alega que el periodismo no es sólo una actividad informativa, sino un cúmulo de valores que incorporan a esa actividad una dimensión ética y que conecta con la responsabilidad que se debe esperar de una profesión llamada a administrar el derecho democrático de la libertad de expresión y de información.

Este interés por conocer la opinión de los profesionales acerca del periodismo ciudadano también ha estado presente en algunos estudios previos desarrollados en otros contextos donde se han hallado resultados similares. Por ejemplo, en la investigación conducida por Önebring (2013) entre periodistas de seis países (Reino Unido, Alemania, Italia, Suecia, Polonia y Estonia), se corrobora esa percepción negativa basada en una serie de elementos diferenciadores y de autoridad respecto a las prácticas amateur, tales como su función de gatekeeping o selección editorial de información relevante, así como la adherencia a valores éticos y deontológicos que aseguran un tratamiento riguroso de los hechos.

Este último aspecto se relaciona, según Örnebring, con un nuevo modo de legitimidad señalado por los periodistas como colectivo, como individuos que pertenecen a organizaciones con valores compartidos y que se diferencian de forma clara de los usuarios, normalmente aislados. Como indica Örnebring (2013: 48): "The straw-man citizen journalist is outside this collective, outside the system of shared knowledge and controls. The total absence in the material of any kind of claim of uniqueness or distinction based on individual autonomy is very striking"

Sería, por tanto, una suerte de sentimiento corporativista que también se refleja en varias de las respuestas obtenidas en la presente investigación.

En otra investigación desarrollada por Blaagaard (2013) se analiza la percepción de estudiantes internacionales de periodismo para obtener resultados similares; es decir, a través de sus respuestas, obtenidas a partir de focus groups, se realiza una diferenciación entre los profesionales del periodismo y los ciudadanos amateur con base en los valores inherentes a la profesión. No obstante, se aporta una dimensión adicional de gran interés; la precariedad laboral de los periodistas relacionada con el surgimiento de webs alternativas de información que no son conducidas por profesionales. Ese temor al intrusismo laboral se establece como una barrera más a la consideración del periodismo ciudadano como tal. 
En un contexto diferente, concretamente el chino, Tong (2015) llega a conclusiones similares tras entrevistar a periodistas del país. Éstos ven a los ciudadanos más como proveedores de material informativo con quienes es posible colaborar esporádicamente, que como competidores en su labor periodística. $Y$ es que en este estudio se vuelve a incidir en la naturaleza corporativa del periodismo, es decir, en la inclusión del periodista en una entidad organizada y con una serie de valores profesionales que marca la diferencia respecto a otras prácticas.

The job trains journalists to produce credible content in a professional way, while organisational guidelines and professional norms guide and limit journalistic practices to ensure they are professional. In this sense, the typical employment relationship between journalists and news organisations is crucial in the boundary work of Chinese journalism and marks out the boundaries of journalism (Tong, 2015: 611).

Se podría establecer, por tanto, la dimensión colectiva del periodismo, ejercido dentro de una estructura empresarial donde priman valores profesionales compartidos, como el principal argumento aportado por los propios periodistas para justificar su percepción general negativa o simplemente diferenciadora respecto al denominado periodismo ciudadano, al cual confieren otros atributos como la provisión puntual de información o cierta interactividad que repercute en una mayor apertura de los medios de comunicación.

\section{Conclusiones}

1. La interactividad se ha convertido en una de las características esenciales del periodismo digital y de la nueva construcción del espacio público, si bien los procesos que se desarrollan en internet son parte de un tejido mucho más grande de la comunicación y la interacción social. No obstante, dicha interactividad ha permitido que el usuario adquiera un cierto grado de control sobre el proceso de difusión de los contenidos y haya aumentado sus posibilidades para dialogar, intercambiar, apoyar y entablar una relación con otros usuarios o con el propio periodista en el marco del medio de comunicación (Suárez-Villegas y Jiménez-Gómez, 2015).

2. El periodismo ciudadano es una práctica que ha hallado su principal capacidad de acción alejado de la gestión profesional, es decir, en formatos descentralizados con una alta capacidad de interacción entre usuarios, como es el caso de los blogs y las redes sociales. Son prácticas que elaboran contenidos de los cuales no se puede presumir tengan 
un valor profesional; es decir, que haya sido elaborado siguiendo un protocolo de diligencia informativa, aunque sí un alto valor social. Por eso, los periodistas han de advertir que aunque ambos actores cumplen funciones distintas y, por tanto, asumen también distintas responsabilidades, en cambio, comparten el mismo objetivo de alcanzar mayores criterios para analizar los acontecimientos.

3. A pesar de la utilización de estos contenidos amateur por parte de los medios de comunicación tradicionales, la etiqueta de periodista ciudadano ha sido objeto de controversia tanto a nivel académico como profesional, al considerarse que el mero hecho de remitir un contenido noticioso no otorga la categoría de periodista a cualquiera. De igual modo, el término periodismo ciudadano también entra en conflicto con otras etiquetas como periodismo interactivo o participativo, que suponen prácticas diferentes de acuerdo al grado de autonomía de ciudadano en la configuración de la información y su relación con el medio, tal y como se establece en la categorización creada por Nip (2006).

4. Este optimismo comunicativo ha resultado ser un espejismo, pues dicha actividad frenética y exuberante por parte de la ciudadanía no ha contribuido necesariamente a establecer una agenda de temas veraces y de interés público. Es por ello que el periodismo ciudadano se ha revelado como un concepto borroso que confunde el ejercicio privado de comunicarse y ofrecer testimonios con la responsabilidad pública de informar, la cual exige una serie de deberes a los periodistas profesionales (Suárez-Villegas y Jiménez-Gómez, 2015).

\section{Referencias}

Anden-Papadopoulos, K. y Pantti, M. (2011), Amateur images and global news, Estados Unidos: Intellect Books.

Blaagaard, B. B. (2013), "Shifting boundaries: Objectivity, citizen journalism and tomorrow's journalists", en Journalism, vol. 14, núm. 8, Reino Unido: Sage Journals.

Bowman, Shayne y Willis, Christ (2003), We Media: How audiences are shaping the future of news and information, Estados Unidos: The Media Center at the American Press Institute, Hypergene. Disponible en: http://www.hypergene.net/ [19 de octubre de 2015].

Bruno, Nicola (2011), "Tweet First, Verify Later: How Real-Time Information is Changing the Coverage of Worldwide Crisis Events", paper for the Reuters Institute for the Study of Journalism, Oxford: University of Oxford. Disponible en: http://reutersinstitute. politics.ox.ac.uk/fileadmin/documents/Publications/fellows_papers/2010-2011/ tweet_first_verify_later.pdf [4 de septiembre de 2015]. 
Bruns, Alex (2008), Blogs, Wikipedia, Second Life and beyond, Estados Unidos: Peter Lang. Dahlgren, P. (2016), "Professional and citizen journalism: Tensions and complements", en Alexander, J. C., Breese, E. B. y Luengo, M. [eds.], The Crisis ofJournalism Reconsidered: Democratic Culture, Professional Codes, Digital Future, Inglaterra: Cambridge University Press.

Gillmor, Dan (2006), We the Media: Grassroots Journalism by the People, for the People, Estados Unidos: O'Reilly.

Harrison, Teresa y Barthel, Brea (2009),"Wielding New Media in Web 2.0: Exploring the History of Engagement with the Collaborative Construction of Media Products", en New Media \& Society, vol. 11, núm. 1-2, Estados Unidos: Sage.

Harro-Loit, H. (2015), “Journalists' Views About Accountability to Different Societal Groups", en Journal of Media Ethics, vol. 30, núm. 1, Estados Unidos: Lawrence Erlbaum Associates.

Hughes, Sallie (2012), "Qualitative supplement of the Worlds of Journalism core questionnaire", en Hanitzsch, T. [coord.], Worlds of journalism. Disponible en: http:// worldsofjournalism.org/download.htm [23 de septiembre de 2015].

Malone, Thomas et al. (2009), "Harnessing Crowds: Mapping the Genome of Collective Intelligence", en MIT Sloan Research Paper, núm. 4732-09. Disponible en: http://ssrn. com/abstract $=1381502$ [30 de octubre de 2015].

Mortensen, T. (2014), "Comparing the Ethics of Citizen Photojournalists and Professional Photojournalists: A Coorientational Study", en Journal of Mass Media Ethics, vol. 29, núm. 1, Estados Unidos: Lawrence Erlbaum Associates.

Nip, Joyce (2006), "Exploring the Second Phase of Public Journalism”, en Journalism Studies, vol. 7, núm. 2, Estados Unidos: Lawrence Erlbaum Associates.

Örnebring, H. (2013), "Anything you can do, I can do better? Professional journalists on citizen journalism in six European countries", en International Communication Gazette, vol. 75, núm. 1, Reino Unido: Sage.

Singer,Jane (2005), “The Marketplace of Ideas-With a Vengeance”, en Media Ethics Magazine, vol. 16, núm. 2, Estados Unidos: University of Illinois at Urbana-Champaign.

Suárez-Villegas, Juan Carlos y Jiménez-Gómez, Eva (2015), "Los periodistas digitales españoles ante la participación ciudadana: ¿Hacia una mejor democracia?, en R. Cetina et al. [eds.], Medios y Periodistas en la era del Gobierno abierto y la transparencia, España: Latin Cyberlaw Clinic, Universidad Complutense y CIEDI. Disponible en: http://eprints.ucm.es/31385/1/Media\%20and\%20journalists\%20in\%20the\%20 age $\% 20$ of $\% 20$ Open $\% 20$ Government $\% 20$ and $\% 20$ Transparency\%20Medios $\% 20$ y\%20periodistas\%20en\%20la\%20era\%20del\%20gobierno\%20abierto\%20y\%20 la\%20tr.pdf [8 de septiembre de 2015].

Tong, J. (2015), "Chinese journalists' views of user-generated content producers and journalism: a case study of the boundary work of journalism", en Asian Journal of Communication, vol. 25, núm. 6, Singapur: Sage. 
Juan Carlos Suárez-Villegas. Doctor en Filosofía. Profesor titular de la Universidad de Sevilla, España. Principales líneas de investigación: ética y deontología del periodismo; igualdad de género; interculturalidad. Publicaciones recientes: Suárez-Villegas, J. C., "Selfregulation of offline and online journalism in Spain in the experience of the Arbitration, Complaints and Ethics Commission”, en Communication \& Society, vol. 28, núm. 3 (2015); Suárez-Villegas, J. C y Cruz-Álvarez, J., "Cambios en los patrones de consumo entre los estudiantes de periodismo: de prosumidores mediáticos a profesionales de la información”, en Estudios sobre el mensaje periodistico, vol. 21, núm. 1, enero-junio (2015); Suárez-Villegas, Juan-Carlos, “Nuevas tecnologías y deontología periodística: comparación entre medios tradicionales y nativos digitales", en El profesional de la información, vol. 24, núm. 4. Disponible en: http://dx.doi.org/10.3145/epi.2015.jul.05 (2015).

Recepción: 27 de octubre de 2015.

Aprobación: 24 de octubre de 2016. 
